\title{
Flow-Induced Structure and Viscoelastic Properties of Poly(styrene-block-2-vinylpyridine)s Solutions near the Order-Disorder Transition
}

\author{
Yoshiaki TAKAHASHI, ${ }^{1, \dagger, \dagger \dagger}$ Masahiro NOdA, ${ }^{2}$ Shinichi KitAde, ${ }^{2}$ \\ Kouki MatsuOKA, ${ }^{2}$ Yushu Matsushita, ${ }^{2}$ and Ichiro NODA ${ }^{2}$ \\ ${ }^{1}$ Center for Integrated Research in Science and Engineering, Nagoya University, \\ Furo-cho, Chikusa-ku, Nagoya 464-8603, Japan \\ ${ }^{2}$ Department of Applied Chemistry, Nagoya University, Furo-cho, Chikusa-ku, Nagoya 464-8603, Japan
}

(Received May 16, 2005; Accepted August 15, 2005; Published December 15, 2005)

\begin{abstract}
Flow effects on the structure and the related viscoelastic properties of lamellar forming poly(styreneblock-2-vinylpyridine)s (SP) and DP in which S in SP replaced by deutrated chains are studied by small angle neutron scattering (SANS) and viscoelastic measurements under shear flows near the order-disorder transition (ODT). A distinctive feature of this sample is that both components have quite similar viscoelastic properties. In the quiescent ordered states, SANS intensities showed strong anisotropy denoting flow-induced alignment of lamellar structure at high shear rates $(\dot{\gamma})$ in a limited range of concentration. When the lamellae are well aligned, first normal stress difference was proportional to $\dot{\gamma}^{2}$ and reduced steady state compliance $J_{\mathrm{eR}}$ became practically the same as those of components. The viscosity behaviors in the quiescent ordered and disordered states were also the same as those of components. In the disordered states, on the other hand, $J_{\mathrm{eR}}$ was higher than those of components. Under steady shear flows, however, SANS intensities are isotropic and became lower and $J_{\mathrm{eR}}$ became almost the same as those of components denoting the suppression of fluctuation effects. Because of the similar viscoelastic properties of components, SPs may flow uniformly compared to other block copolymers such as poly(styrene-block-isoprene)s. [DOI 10.1295/polymj.37.894] KEY WORDS Poly(styrene-block-2-vinylpyridine)s / Flow-Induced Structure / Viscoelastic Properties / Lamellar Structure / Small Angle Neutron Scattering /
\end{abstract}

Block copolymers possess different microphaseseparated structure in the ordered states, such as sphere, cylinder, co-continuous and lamella, depending on the molecular structure and compositions of components. In the disordered states but near the order-disorder transition (ODT), there exist composition fluctuations of component polymers in the block copolymer liquids. Both the microphase separated structure and the composition fluctuations affect the rheological properties of block copolymers, therefore, structure and the related viscoelastic properties of the block copolymers have been extensively studied both in the ordered and the disordered states. General results of rheological properties for variety of block copolymers are summarized in review articles. ${ }^{1,2}$ Here, we briefly mention the characteristic features of structure and viscoelastic properties for the lamellar forming diblocks, which are closely related to this study.

In the quiescent ordered states, storage, $G^{\prime}(\omega)$, and loss, $G^{\prime \prime}(\omega)$, moduli measured with small strains show power law dependence on frequency $\omega\left(G^{\prime}(\omega)\right.$, $\left.G^{\prime \prime}(\omega) \sim \omega^{1 / 2}\right)$ at the low $\omega$ end due to the large scale motions of randomly oriented grains separated by defects. When the structure is well aligned, the contribu- tions from multi grain structure on $G^{\prime}$ and $G^{\prime \prime}$ become insensitive and molecular responses become apparent so that the $G^{\prime}$ and $G^{\prime \prime}$ values in the power law region become smaller. Under steady shear flow, moreover, shear rate dependence of first normal stress difference $N_{1}$ changes from $\dot{\gamma}$ to $\dot{\gamma}^{2}$ when the lamellae are well aligned. $^{3}$

In the quiescent disordered states, it was reported that the fluctuation effects are more pronounced on the viscoelastic properties than those on scattering data. ${ }^{4}$ Both $G^{\prime}$ and $G^{\prime \prime}$ are enhanced due to the fluctuation effects, which was more obvious for $G^{\prime}$ than $G^{\prime \prime} .5,6$ The fluctuation effects become smaller for higher molecular weight samples. ${ }^{5}$ Moreover, flow induced ordering was reported in the quiescent disordered states close to the ODT. ${ }^{7-9}$

In previous papers, ${ }^{10-13}$ we studied order-disorder transition (ODT) ${ }^{10}$ viscosity behaviors in the disordered states ${ }^{11}$ and flow induced structure in the ordered states ${ }^{12,13}$ for symmetric poly(styrene-block-2vinylpyridine)s (SP) and DP, in which $\mathrm{S}$ in SP replaced by deutrated polystyrene block. Distinctive features of SPs (and DPs) compared with other block copolymers such as poly(styrene-block-isoprene) (SI)

\footnotetext{
${ }^{\dagger}$ Present Address: Department of Molecular \& Materials Sciences, Kyushu University, 6-1 Kasugakoen, Kasuga 816-8580, Japan

${ }^{\dagger}$ To whom correspondence should be addressed (Tel/Fax: +81-92-583-8822, E-mail: ytak@mm.kyushu-u.ac.jp).
} 
Table I. Molecular characteristics of poly(styrene- $b$-2-vinylpyridine)s

\begin{tabular}{lcccc}
\hline $\begin{array}{l}\text { Sample } \\
\text { Code }\end{array}$ & $10^{-4} M_{\mathrm{w}}$ & $\begin{array}{c}M_{\mathrm{w}} / M_{\mathrm{n}} \\
(\text { by GPC) }\end{array}$ & $\phi_{\mathrm{PS}}$ & $\begin{array}{c}C_{\mathrm{ODT}^{\mathrm{a}}} \\
\left(\mathrm{kg} / \mathrm{dm}^{3}\right)\end{array}$ \\
\hline SP23 & 109 & 1.03 & 0.53 & 7.4 \\
SP12 & 42.2 & 1.03 & 0.52 & 12.7 \\
DP20 & 36.0 & 1.08 & 0.53 & 13.9 \\
DP19 & 21.0 & 1.05 & 0.52 & 20.0 \\
SP22 & 3.8 & 1.01 & 0.52 & 48.8 \\
SP26 & 1.1 & 1.02 & 0.51 & - \\
\hline
\end{tabular}

${ }^{\mathrm{a}}$ at $30^{\circ} \mathrm{C}$.

are that both component polymers, polystyrene (PS) and poly(2-vinylpiridyne) (P2VP) have almost the same Kuhn segment length ${ }^{14}$ and quite similar viscoelastic properties at melt states and in common good solvents. ${ }^{15}$ In contrast to the results mentioned above, flow induced alignment of lamellar structure for DPs (DP19 and DP20; see Table I) in a common good solvent in the ordered states was observed only at limited conditions. ${ }^{12,13}$ That is, flow induced alignment did not occur for a low molecular weight sample, DP19, even at the highest shear rate tested $\left(1000 \mathrm{~s}^{-1}\right)$ in the small-angle neutron scattering measurements under the steady shear flows (flow-SANS). The flow induced alignment was observed for a higher molecular weight sample, DP20, at concentrations few \% higher than ODT, but the alignment became very weak with slight decrease of concentration or slight increase of temperature.

In this work, we report further studies of flow effects on the structure examined by flow-SANS and related viscoelastic properties of symmetric SP and DP solutions in a common good solvent above and below the ODT. In the ordered states, viscoelastic properties of aligned systems are directly compared with the data for the components. In the disordered states, flow effects on the composition fluctuations are examined by dynamic and steady shear flow measurements.

\section{EXPERIMENTAL}

Block copolymer samples used are the same SPs and DPs as used in the previous studies. ${ }^{10-14}$ Molecular characteristics of the samples $\left(M_{\mathrm{W}}\right.$ : weight averaged molecular weight, $M_{\mathrm{n}}$ : number averaged molecular weight, $\phi_{\mathrm{PS}}$ : volume fraction of styrene) including ODT concentrations, $C_{\text {ODT }}$ determined by dynamic mechanical analysis and ordinary SANS measurements ${ }^{10}$ are tabulated in Table I. A common good solvent used was $\alpha$-chloronaphtharene $(\alpha \mathrm{CN})$, which is also the same as in previous works. Note that polymer concentration $C$ is presented by $\mathrm{kg} / \mathrm{dm}^{3}(=\mathrm{g} / \mathrm{dL})$ to easily compare with earlier studies unless otherwise specified.
Viscoelastic properties of SP and DP solutions are measured with a Mechanical Spectrometer RMS800 and a high sensitivity version of Ares (FS type) of Rheometric Scientific. A cone-plate geometry of $5 \mathrm{~cm}$ diameter and 0.04 rad cone-angle was used. The solutions in the quiescent ordered states are mainly measured under steady shear flow. The solutions in the quiescent disordered states are mainly measured under small amplitude oscillatory shear flow. In addition, a few solutions are also measured under the steady shear flow. Most of the rheological measurements are carried out at $30.0 \pm 0.5^{\circ} \mathrm{C}$. A few viscosity data for SP23 in this work and DP19 in a previous paper ${ }^{10}$ are measured at slightly lower temperatures. The difference in temperature was reduced by using specific viscosity and reduced steady state compliance.

Flow-SANS measurements are performed using a couette type flow cell, ${ }^{16}$ attached to the SANS-U spectrometer at the Neutron Scattering Laboratory of the ISSP, The University of Tokyo in JAERI Tokai, Japan. An aluminum stator and a quartz rotor, having $0.75 \mathrm{~mm}$ gap are used. The wavelength $\lambda$ and the diameter of incident neutron beam are $0.7 \mathrm{~nm}$ and $3 \mathrm{~mm}$, respectively. The sample to detector distances are 8 and $12 \mathrm{~m}$. Two concentrations, 16.2 and $13.0 \mathrm{~kg} /$ $\mathrm{dm}^{3}$, in the ordered and the disordered states, respectively are examined. Two types of measurements, that is, "through view," in which the incident neutron beam is in the direction normal to the surface of the center part of couette cell (incident beam perpendicular to the flow) and "edge (tangential) view" measurement, in which the incident beam going through the edge of cell (incident beam parallel to the flow) are employed. The data obtained by the through view measurements are corrected for the incoherent scattering of the solvent and the empty cell. As already reported, ${ }^{2,13}$ lamellar normal of DP20 solutions are preferentially aligned to the vorticity direction of the steady shear flow, called perpendicular alignment, when alignment occur. In that case, the data are discussed as averaged intensities within sectors of $\pm 5^{\circ}$ arc in horizontal $\left(I_{\mathrm{X}}\right)$ and perpendicular $\left(I_{\mathrm{Y}}\right)$ directions on the detector as a function of the magnitude of wave vector, $q$, defined by $q=4 \pi \sin (\theta / 2) / \lambda$, where $\theta$ is scattering angle. Otherwise, circularly averaged intensity $I$ is used. The edge view measurements are only used to confirm the flow-induced structure. The measurements are carried out at room temperature $\left(c a .27^{\circ} \mathrm{C}\right)$.

\section{RESULTS}

Figure 1 show double logarithmic plots of $G^{\prime}$ and $G^{\prime \prime}$ against $\omega$ in the quiescent disordered states. A set of data in the quiescent ordered states are shown for comparison. It is apparent that the data obtained 


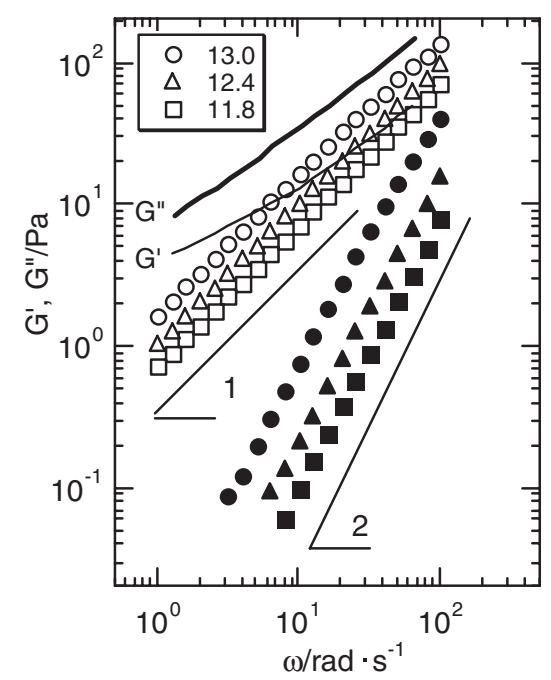

Figure 1. Double logarithmic plots of $G^{\prime}$ and $G^{\prime \prime}$ against $\omega$ in the quiescent disordered states. Sample is DP20 and the concentrations $\left(\mathrm{kg} / \mathrm{dm}^{3}=\mathrm{g} / \mathrm{dL}\right)$ are denoted in the figure. The closed and open symbols denote $G^{\prime}$ and $G^{\prime \prime}$, respectively. Solid and broken lines represent $G^{\prime}$ and $G^{\prime \prime}$ data in the ordered states $(C=$ 16.2), respectively.

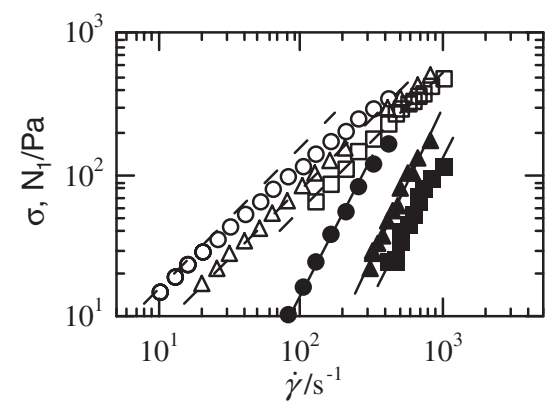

Figure 2. Double logarithmic plots of $\sigma$ (open symbols) and $N_{1}$ (closed symbols) against $\dot{\gamma}$ in the quiescent disordered states. The symbols are the same as Figure 1. Broken and solid lines denote the slope of 1 and 2, respectively.

in the disordered states show the terminal region behavior. The terminal region behavior is also observed for other samples in the disordered states. Figure 2 show double logarithmic plots of shear stress $\sigma$ and first normal stress difference $N_{1}$ against shear rate $\dot{\gamma}$ for the same solutions in Figure 1. The linear viscoelastic behaviors are clearly observed in Figure 2. Zero shear viscosity $\eta^{0}$ and steady state compliance $J_{\mathrm{e}}$ are evaluated from these data by the ordinary methods, ${ }^{17}$ which will be discussed later.

Figure 3 show $\dot{\gamma}$ dependences of $\sigma$ and $N_{1}$ in the ordered states for SP12 and DP20 solutions. It is clear in Figure 3a that all $\sigma$ data are proportional to $\dot{\gamma}$ in wide ranges of shear rate, while deviation from the proportionality is observed for some solutions at high shear rates. There is no practical difference between the shear rate dependence of $\sigma$ in the disordered
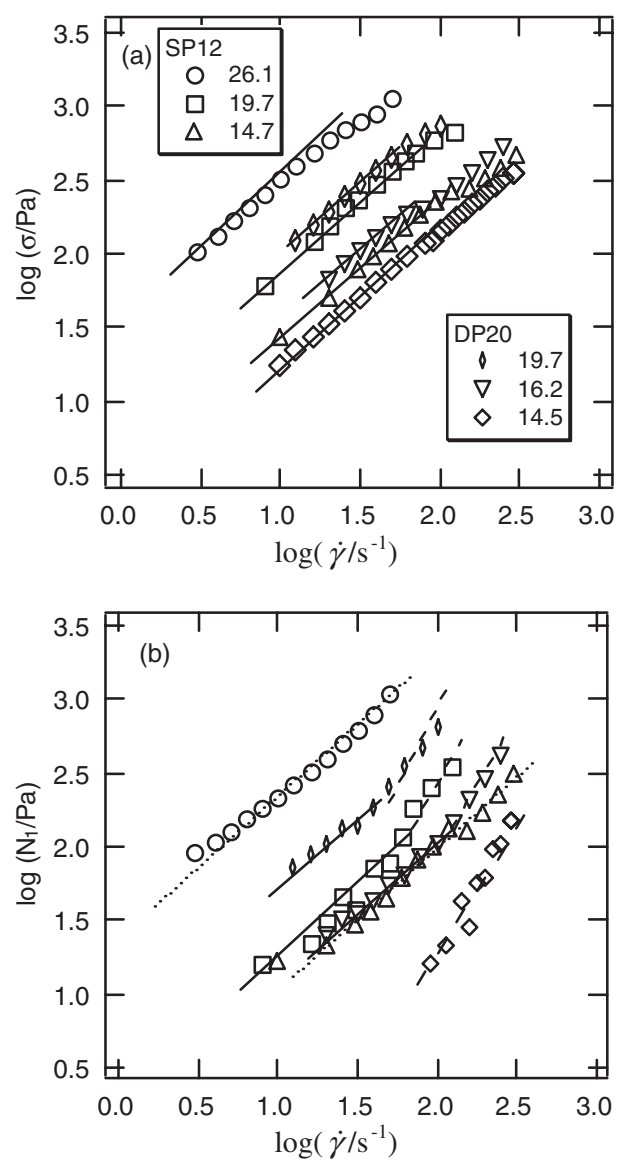

Figure 3. Examples of double logarithmic plots of $\sigma$ and $N_{1}$ against $\dot{\gamma}$ in the quiescent ordered states. Symbols are denoted in the figure. Dotted and solid lines denote the slope of 1 and 2, respectively. The broken line in (b) is guide for eyes showing slightly lower shear rate dependence than those of well aligned systems.

(Figure 2) and ordered states (Figure 3a). In contrast, apparent differences in the $\dot{\gamma}$ dependence of $N_{1}$ are observed for different samples and concentrations. The $\dot{\gamma}$ dependence of $N_{1}$ was slightly lower than $\dot{\gamma}^{2}$ for the lowest concentration of DP20 as guided by a broken line in Figure 3b. $N_{1}$ was almost proportional to $\dot{\gamma}$ in the entire $\dot{\gamma}$ range for the highest and the lowest concentrations of SP12. For other concentrations, $N_{1}$ is proportional to $\dot{\gamma}$ at low $\dot{\gamma}$ range, but it becomes proportional to $\dot{\gamma}^{2}$ at higher $\dot{\gamma}$ range. This behavior is qualitatively the same as that reported for a SI/DOP solution in the ordered states. ${ }^{3}$ High $M_{\mathrm{w}}$ sample, SP23 also showed qualitatively the same behavior, while $N_{1}$ of low $M_{\mathrm{w}}$ samples are almost proportional to $\dot{\gamma}$ in the whole experimental range of shear rate in the ordered states.

Figure 4 shows semilogarithmic plots of $I_{Y}$ against $q$ for $16.2 \mathrm{~kg} / \mathrm{dm}^{3}$ solution. It is clear that the peak intensity significantly increase and higher order peaks appear at relative $q$ positions of 1:2:3, indicating perpendicular alignment of lamellar structure under the 


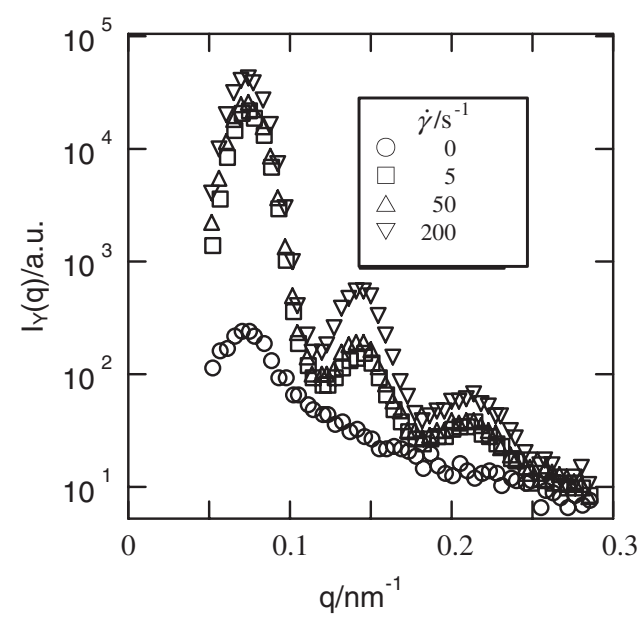

Figure 4. Plots of sector averaged SANS intensity $I_{\mathrm{Y}} v s . q$ at different shear rates in the quiescent ordered state $\left(16.2 \mathrm{~kg} / \mathrm{dm}^{3}\right)$. Shear rates are denoted in the figure.

flow. The data for 5 and $50 \mathrm{~s}^{-1}$ are not so much different with each other. At $200 \mathrm{~s}^{-1}$ peak intensities become higher than those at lower shear rates especially for the higher order peaks, implying that the degree of alignment is much higher at this shear rate. Note that $N_{1}$ was proportional to $\dot{\gamma}$ at 5 and $50 \mathrm{~s}^{-1}$, while it was proportional to $\dot{\gamma}^{2}$ at $200 \mathrm{~s}^{-1}$. As reported previously, ${ }^{12}$ the data for almost the same concentration but slightly different temperatures are qualitatively the same as the data in this figure. On the other hand, much weak alignment was observed for $14.7 \mathrm{~kg} / \mathrm{dm}^{3}$ solution..$^{13}$ The $I_{\mathrm{Y}} / I_{\mathrm{X}}$ value exceeds 100 for $16.2 \mathrm{~kg} /$ $\mathrm{dm}^{3}$ at $200 \mathrm{~s}^{-1}$ (this work), while it was about 10 for $14.7 \mathrm{~kg} / \mathrm{dm}^{3}$ at $400 \mathrm{~s}^{-1}$ (ref 13), implying that the structure is only slightly aligned under the flow for concentrations very close to ODT. As shown in Figure 2, shear rate dependence of $N_{1}$ at $14.5 \mathrm{~kg} /$ $\mathrm{dm}^{3}$ was somewhat different from those of the solutions with higher concentrations. This behavior can be attributed to the weak alignment of the structure mentioned above.

The flow-SANS data for $13.0 \mathrm{~kg} / \mathrm{dm}^{3}$ solution (disordered state) was always isotropic even at the highest shear rate $\left(360 \mathrm{~s}^{-1}\right)$ both in through view and edge view. Figure 5 compares $I(q)$ at rest and those under the flow for $13.0 \mathrm{~kg} / \mathrm{dm}^{3}$ solution. The shape of $I(q)$ curves are almost identical, indicating that the solution is also in the disordered states under the flow. The peak intensity becomes slightly lower with increase of shear rate implying that the fluctuation effects are suppressed by the flow.

\section{DISCUSSIONS}

As shown in Figure $3 \mathrm{a}, \sigma$ are proportional to $\dot{\gamma}$ at low shear rates so that we can obtain $\eta^{0}$ in the

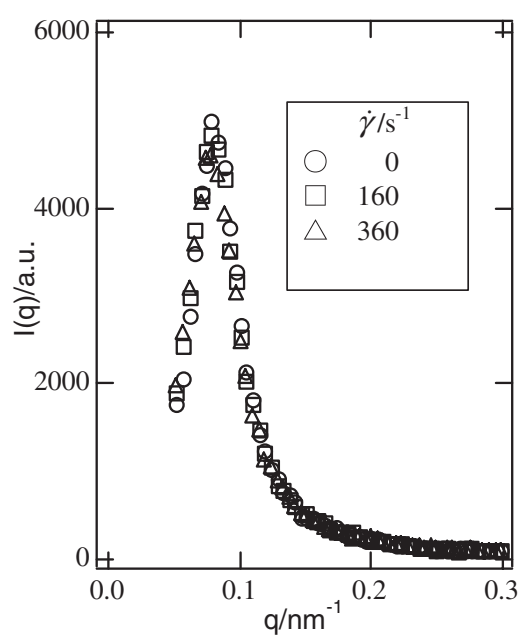

Figure 5. Plots of circularly averaged SANS intensity $I$ vs. $q$ at different shear rates in the quiescent disordered state $(13.0 \mathrm{~kg} /$ $\mathrm{dm}^{3}$ ). Shear rates are denoted in the figure.

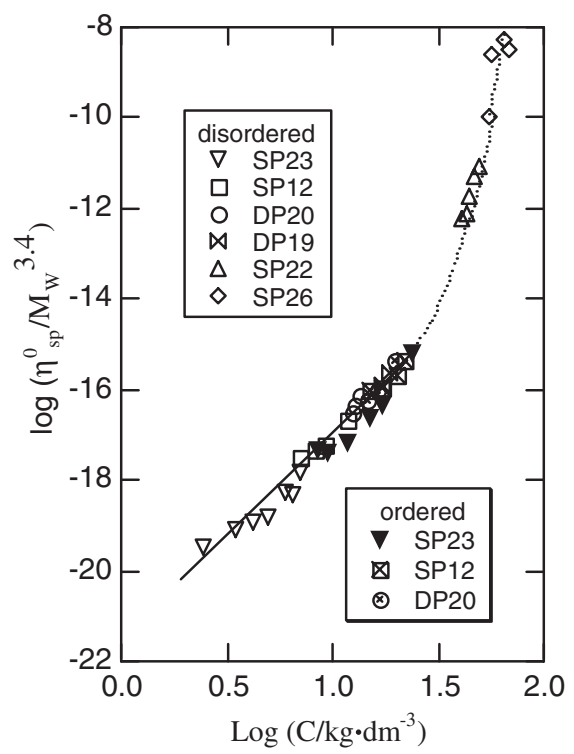

Figure 6. Plots of $\log \left(\eta_{\mathrm{sp}}^{0} / M_{\mathrm{W}}^{3.4}\right)$ against $\log C$ for SP diblock copolymers in $\alpha \mathrm{CN}$. Symbols are represented in the figure. Solid and dotted lines denote the data for $\mathrm{PS}^{6}$ and $\mathrm{P}_{2} \mathrm{VP}^{12}$ in semidilute and concentrated regions, respectively.

quiescent ordered states. When apparent flow-induced alignment occurs, shear rate dependence of $N_{1}$ become proportional to $\dot{\gamma}^{2}$, so that we can obtain $J_{\mathrm{e}}$ of the aligned samples. We compare the $\eta^{0}$ and $J_{\mathrm{e}}$ data in the quiescent ordered states with those obtained in the disordered states and those of component homopolymers. ${ }^{15,17,18}$ The viscosity data obtained from oscillatory and steady shear measurements in the disordered states are practically the same, so that the only former are discussed here.

Figure 6 shows double logarithmic plots of specific viscosity $\eta_{\mathrm{sp}}^{0}$ reduced by the molecular weight dependence $\left(M_{\mathrm{W}}{ }^{3.4}\right)$ in the entangled region against polymer concentration $C$. Here, $\eta_{\mathrm{sp}}^{0}$ is defined as $\eta_{\mathrm{sp}}^{0}=\left(\eta^{0}-\right.$ 
$\left.\eta_{\mathrm{s}}\right) / \eta_{\mathrm{s}}, \eta_{\mathrm{s}}$ being the solvent viscosity. The data for component homopolymers ${ }^{15,18}$ in semidilute $(C<20$ $\left.\mathrm{kg} / \mathrm{dm}^{3}\right)$ and concentrated regions $\left(C>20 \mathrm{~kg} / \mathrm{dm}^{3}\right)$ are also shown by solid and dotted lines, respectively. It is clear that all the data compose a single line irrespective of the molecular weights and coincide with those of homopolymers. Therefore, it is concluded that the viscosity behavior of SP and DP diblock copolymers in common good solvents are the same as those of component polymers both in the ordered and disordered states. Unlike to the results of other block copolymers, ${ }^{1,4,6}$ contributions from multi grain structure to the viscosity are not obviously observed under steady shear flow in the ordered states. Enhancement of viscosity due to the fluctuation effect in the disordered states has not been detected even at the concentrations very close to the ODT.

Figure 7 shows double logarithmic plots of $J_{\mathrm{eR}} v s$. $C M_{\mathrm{W}}$, where the reduced steady state compliance $J_{\mathrm{eR}}$ is defined by $\left(J_{\mathrm{e}} C \mathrm{R} T / M_{\mathrm{W}}\right)\left[\eta^{0} /\left(\eta^{0}-\eta_{\mathrm{s}}\right)\right]^{2}$. In this figure, the unit of $C$ is set as $10^{3} \mathrm{~kg} / \mathrm{m}^{3}\left(=\mathrm{g} / \mathrm{cm}^{3}\right)$ to easily compare with the data in literature. In Figure $7 \mathrm{~b}$, the data of the aligned samples fall in between two solid lines, denoting the mean values of the data for two component homopolymers. ${ }^{15,18}$ Thus, we conclude that the viscoelastic properties of aligned SP and
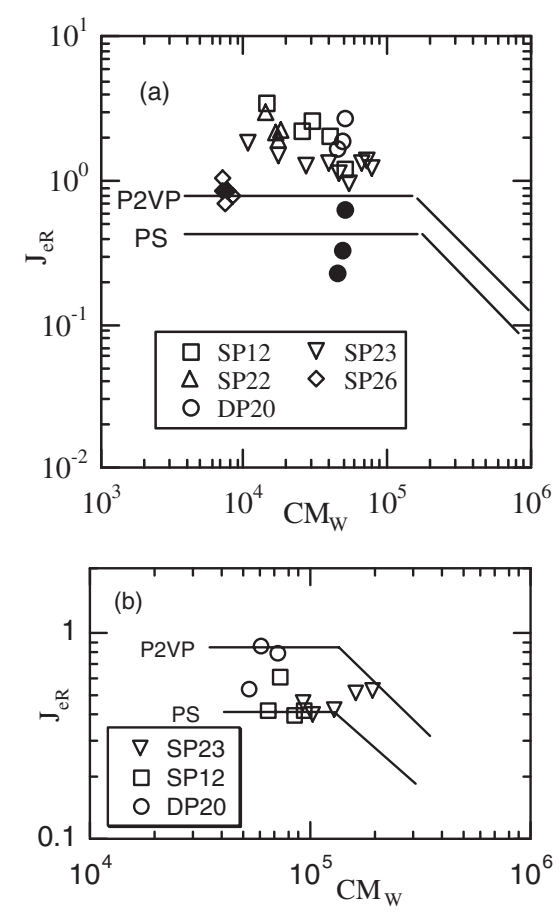

Figure 7. Double logarithmic plots of $J_{\mathrm{eR}}$ against $C M_{\mathrm{W}}$ for SP and DP solutions (a): in the disordered states and (b): in the ordered states. Samples are denoted in the figure. The open and filled symbols in (a) denote the data obtained by oscillatory and steady shear measurements, respectively. Solid lines denote the the data for $\mathrm{PS}^{6}$ and $\mathrm{P} 2 \mathrm{VP}^{12}$, respectively. The unit of $C$ is set as $10^{3} \mathrm{~kg} / \mathrm{m}^{3}(=\mathrm{g} / \mathrm{mL})$.
DP samples are practically the same as those for component polymers.

Figure 7a shows the data obtained for the solutions in the disordered states. Open and filled symbols denote the data obtained by oscillatory and steady flow measurements, respectively. It is clear that most of the data obtained by oscillatory flow are higher than those of components, while the data obtained by steady shear flow for DP20 are lower than those obtained by oscillatory flow and close to the values of components. As shown in Figure 4, the SANS data of DP20 solution in the disordered states was suppressed under the flow, the decrease in $J_{\mathrm{eR}}$ can be attributed to the suppression of the fluctuation effects by steady shear flow, though there was no apparent fluctuation effects in viscosity behavior. As already mentioned, it was reported ${ }^{5,6}$ that the fluctuation effects more strongly affect $G^{\prime}$ than $G^{\prime \prime}$ and the fluctuation effects are more pronounced on the viscoelastic properties than those on scattering data. ${ }^{4}$ All the characteristics of $\eta_{\mathrm{sp}}^{0}, J_{\mathrm{eR}}$ and flow-SANS data mentioned above are qualitatively consistent with those results.

The data obtained for SP26 in the two different flows are almost the same and fall into the data range of homopolymers. ODT of this sample is not determined but it is estimated ${ }^{10}$ to be close to bulk state even at $30^{\circ} \mathrm{C}$. Therefore, essentially no fluctuation effects are expected for this sample. Thus, it is natural that there was no practical difference between the data obtained by oscillatory and steady shear flows.

There may be a few possible reasons for difference in flow-induced phenomena of the block copolymers studied in this work and those of other studies. One possibility is the difference in the concentrationmolecular weight relationships of ODT points. However, this may not be the main reason, since the relationship between ODT concentrations and degree of polymerizations for DPs or $\mathrm{SPs}^{10}$ is quite similar to that of SI's ${ }^{19}$ at constant temperature. There still exists a possibility that the magnitudes and frequency of fluctuations are different for different polymer species, even the molecular weight and concentration dependences of ODT are similar.

We speculate that the main reason for the difference is in the difference of viscoelastic properties of components. Usually, viscoelastic properties of component blocks are different so that there is a periodic contrast of viscoelastic properties in the ordered states. When flow is applied, each component may show different responses to the flow, resulting in the most favorite orientation to minimize the resistance to the flow. Similar contrast of viscoelastic properties also exist in the disordered states governed by fluctuations, which will be the origin of flow-induced microphase separation. The magnitude and frequency 
of fluctuation may also depend on viscoelastic properties of components. In the case of SPs and DPs, on the other hand, they flow uniformly, insensitive to the fluctuations, compared to the other block copolymers since the viscoelastic properties of components are almost the same. Fredrickson ${ }^{20}$ theoretically analyzed the effect of viscosity ratio of components on the flow-induced alignments of diblock copolymers and proposed schematic phase diagram. A more detailed comparison between flow-induced structure of DPs and poly(styrene-block-isoprene)s near the ODT based on the measured interaction parameters and viscosity ratio of components referring the theory will be reported in a separate paper.

\section{CONCLUSIONS}

Flow effects on the structure and related viscoelastic properties of lamellar forming diblock copolymers composed of components having almost the same viscoelasticity are studied by flow-SANS and rheometry near the ODT. In the quiescent ordered states, SANS intensities showed strong anisotropy denoting flowinduced alignment of lamellar structure at high $\dot{\gamma}$ in a limited range of concentration. First normal stress difference $N_{1}$ was proportional to $\dot{\gamma}$ when the alignment is weak but became proportional to $\dot{\gamma}^{2}$ when the lamellar structure was well aligned. The viscoelastic properties of the systems with well aligned structure became practically the same as those of components. The viscosity behavior in the quiescent disordered states was also the same as those of components. However, enhancement of elastic behavior was observed under the oscillatory flow measurements. That is, reduced steady state compliance $J_{\mathrm{eR}}$ was higher than those of components. Under steady shear flows, however, SANS intensities were isotropic and became lower. The values of $J_{\mathrm{eR}}$ became almost the same as those of components. These behaviors can be explained by the suppression of fluctuation effects. It was concluded that these characteristic behaviors of poly(styrene-block-2-vinylpyridine)s are due to the viscoelastic properties of component polymers; both components have quite similar viscoelastic properties so that they flow uniformly compared to other block copolymers.
Acknowledgment. This work was partly supported by a Grant-in-Aid for Scientific Research on Priority Areas (A) (\#13031041-00) from Ministry of Education, Culture, Sports, Science and Technology.

\section{REFERENCES}

1. H. Watanabe, in "Structure and Properties of Multiphase Polymeric Materials," T. Araki, T. C. Qui, and M. Shibayama, Ed., Marcel Dekker, New York, 1998, chapt. 9.

2. I. W. Hamley, Curr. Opin. Collid Interface Sci., 5, 342 (2000).

3. Y. Takahashi, M. Noda, N. Ochiai, and I. Noda, Polymer, 37, 5943 (1996).

4. F. S. Bates, J. H. Rosedale, and G. H. Fredrickson, J. Chem. Phys., 92, 6255 (1990).

5. F. S. Bates, Macromolecules, 17, 2607 (1984).

6. X. Jin and T. P. Lodge, Rheol. Acta, 36, 229 (1997).

7. K. A. Koppi, M. Tirrell, and F. S. Bates, Phys. Rev. Lett., 70, 1449 (1993).

8. N. P. Balsara, B. Hammouda, P. K. Kesani, S. V. Jonnalagadda, and G. C. Straty, Macromolecules, 27, 2566 (1994).

9. N. P. Balsara, H. J. Dai, P. K. Kesani, B. A. Garetz, and B. Hammouda, Macromolecules, 27, 7406 (1994).

10. Y. Takahashi, S. Kitade, M. Noda, N. Ochiai, I. Noda, M. Imai, and Y. Matsushita, Polym. J., 30, 388 (1998).

11. M. Yamaguchi, N. Maeda, Y. Takahashi, M. Matsushita, and I. Noda, Polym. J., 23, 227 (1991).

12. S. Kitade, N. Ochiai, Y. Takahashi, I. Noda, Y. Matsushita, A. Karim, A. I. Nakatani, H. Kim, and C. C. Han, Macromolecules, 31, 8083 (1998).

13. Y. Takahashi, M. Noda, S. Kitade, and I. Noda, J. Phys. Chem. Solids, 60, 1343 (1999).

14. Y. Matsushita, K. Shimizu, Y. Nakao, R. Saguchi, H. Choshi, and M. Nagasawa, Polym. J., 18, 361 (1986).

15. Y. Takahashi, N. Ochiai, Y. Matsushita, and I. Noda, Polym. J., 28, 1065 (1996).

16. Y. Takahashi, M. Noda, M. Naruse, T. Kanaya, H. Watanabe, T. Kato, M. Imai, and Y. Matsushita, Nihon Reoroji Gakkaishi, 28, 187 (2000).

17. W. W. Graessley, Adv. Polym. Sci., 16, 1 (1974).

18. Y. Takahashi, I. Noda, and M. Nagasawa, Macromolecules, 18, 2220 (1985).

19. T. P. Lodge, C. Pan, X. Jin, Z. Liu, J. Zhao, W. W. Maurer, and F. S. Bates, J. Polym. Sci., Part B: Polym. Phys., 33, 2289 (1995).

20. G. H. Fredrickson, J. Rheol., 38, 1045 (1994). 